\title{
Common Fixed Point Theorems in $b$-Metric Spaces
}

\author{
Yan Hao, Hongyan Guan* \\ School of Mathematics and Systems Science, Shenyang Normal University, Shenyang 110034, China \\ *Corresponding author: guanhy8010@163.com
}

Received June 03, 2019; Revised July 06, 2019; Accepted August 07, 2019

\begin{abstract}
In this paper, we prove some common fixed point results for two mappings satisfying contraction conditions in complete $b$-metric spaces. Meanwhile, two examples are presented to support our results.
\end{abstract}

2010 Mathematics Subject Classification. Primary 47H10.

Keywords: common fixed point, b-metric space, contraction condition.

Cite This Article: Yan Hao, and Hongyan Guan, “Common Fixed Point Theorems in b-Metric Spaces.” Turkish Journal of Analysis and Number Theory, vol. 7, no. 4 (2019): 117-123. doi: 10.12691/tjant-7-4-4.

\section{Introduction}

In 1922, Banach [1] proved the Banach contraction principle. Since then, several works have been done about fixed point theory regarding different classes of contractive conditions in some spaces such as: quasi-metric spaces [2,3], cone metric spaces [4,5], partially order metric spaces $[6,7,8]$, G-metric spaces [9].

The concept of $b$ - metric space was introduced by Czerwik in [10]. After that, several papers have been published on the fixed point theory of various classes of single-valued and multi-valued operators in $b$ - metric spaces (see [2,11,12]). Aydi et al. in [13] proved common fixed point results for single-valued and multi-valued mappings satisfying a weak $\phi$-contraction in $b$-metric spaces. Starting from the results of Berinde [14], Pacurar [15] proved the existence and uniqueness of fixed point of $\phi$-contractions on $b$ - metric spaces. Using a contraction condition defined by means of a comparison function, [16] established results regarding the common fixed points of two mappings. Hussain and Shah in [17] introduced the notion of a cone $b$ - metric spaces, generalizing both the notions of $b$ - metric spaces and cone metric spaces, they considered topological properties of cone $b$-metric spaces and results on KKM mappings in the setting of cone $b$ - metric spaces.

The aim of this paper is to consider and establish some common fixed point results for two mappings satisfying contraction conditions in complete $b$-metric spaces. Meanwhile, two examples are presented to support our results.

\section{Preliminaries}

Let $\mathbb{R}$ and $\mathbb{R}^{+}$denote the sets of all real numbers and nonnegative numbers respectively. $N$ denotes the set of positive integers and $N_{0}=N \bigcup\{0\}$. Suppose

$\Phi=\left\{\phi \mid \phi:\left(\mathbb{R}^{+}\right)^{7} \rightarrow \mathbb{R}^{+}\right.$is upper semicontinuous and nondecreasing in each coordinate variable satisfying condition $\phi(t, t, t, t, t, t, t)=\bar{\phi}(t)<t\}$

and

$\Psi=\left\{\psi \mid \psi:\left(\mathbb{R}^{+}\right)^{9} \rightarrow \mathbb{R}^{+}\right.$is upper semicontinuous and nondecreasing in each coordinate variable satisfying condition $\psi(t, t, t, t, t, t, t, t, t)=\bar{\psi}(t)<t\}$.

In order to obtain our main results, we need to introduce some definitions and lemmas.

Definition. Let $X$ be a nonempty set and $d: X \times X \rightarrow[0,+\infty)$. A function $d$ is called a $b-$ metric with constant $s \geq 1$ if

(1) $d(x, y)=0$ if and only if $x=y$;

(2) $d(x, y)=d(y, x)$ for all $x, y \in X$;

(3) $d(x, y) \leq s(d(x, z)+d(y, z))$ for all $x, y, z \in X$.

The pair $(X, d)$ is called a $b$-metric space.

It is obvious a $b$-metric space with $s=1$ is a metric space. There are examples of $b$-metric spaces which are not metric spaces. (see [18])

Definition. Let $\left\{x_{n}\right\}$ be a sequence in a $b$-metric space $(X, d)$.

(1) A sequence $\left\{x_{n}\right\}$ is called convergent if and only if there is $x \in X$ such that $d\left(x_{n}, x\right) \rightarrow 0$ when $n \rightarrow+\infty$;

(2) $\left\{x_{n}\right\}$ is a Cauchy sequence if and only if $d\left(x_{n}, x_{m}\right) \rightarrow 0$ when $n, m \rightarrow+\infty$.

As usual, a $b$-metric space is said to be complete if and only if each Cauchy sequence in this space is convergent.

Lemma 2.1. [19] Let $\psi: \mathbb{R}^{+} \rightarrow \mathbb{R}^{+}$be nondecreasing and upper semicontinuous. Then for each $t>0, \psi(t)<t$ if and only if $\lim _{n \rightarrow \infty} \psi^{n}(t)=0$. 


\section{Main Results}

Now we are ready to prove our main results.

Theorem 3.1. Let $(X, d)$ be a complete $b$-metric space with constant $s \geq 1$. Suppose $A$ and $B: X \rightarrow X$ are two mappings and one of them is continuous. If there exists $\phi \in \Phi$ such that

$$
\begin{aligned}
& s^{4} d(A x, B y) \\
& \leq \phi\left(\begin{array}{l}
d(A x, x), d(B y, y), d(x, y), \\
\frac{d(A x, x)+d(B y, y)}{2}, \frac{d(A x, x)+d(x, y)}{2}, \\
\frac{d(B y, y)+d(x, y)}{2}, \frac{d(A x, y)+d(B y, x)}{2}
\end{array}\right)
\end{aligned}
$$

for all $x, y \in X$, then $A$ and $B$ have a unique common fixed point $x^{*} \in X$.

Proof. Let $x_{0} \in X$ be arbitrary. We define a sequence $\left\{x_{n}\right\}$ as follows:

$$
x_{2 n+1}=A x_{2 n}, x_{2 n+2}=B x_{2 n+1}, n \in N .
$$

We now suppose that $d\left(x_{n}, x_{n+1}\right)>0$ for every $n$. If not, there exists some $n \in N$ such that $x_{n}=x_{n+1}$. If $n=2 k$, then $x_{2 k}=x_{2 k+1}$ and from the contraction condition (1) with $x=x_{2 k}$ and $y=x_{2 k+1}$, we have

$$
\begin{aligned}
& s^{4} d\left(x_{2 k+1}, x_{2 k+2}\right)=s^{4} d\left(A x_{2 k}, B x_{2 k+1}\right) \\
& \leq \phi\left(\begin{array}{l}
d\left(A x_{2 k}, x_{2 k}\right), d\left(B x_{2 k+1}, x_{2 k+1}\right), \\
d\left(x_{2 k}, x_{2 k+1}\right), \\
\frac{d\left(A x_{2 k}, x_{2 k}\right)+d\left(B x_{2 k+1}, x_{2 k+1}\right)}{2}, \\
\frac{d\left(A x_{2 k}, x_{2 k}\right)+d\left(x_{2 k}, x_{2 k+1}\right)}{2}, \\
\frac{d\left(B x_{2 k+1}, x_{2 k+1}\right)+d\left(x_{2 k}, x_{2 k+1}\right)}{2}, \\
\frac{d\left(A x_{2 k}, x_{2 k+1}\right)+d\left(B x_{2 k+1}, x_{2 k}\right)}{2}
\end{array}\right) \\
& =\phi\left(\begin{array}{l}
d\left(x_{2 k+1}, x_{2 k}\right), d\left(x_{2 k+2}, x_{2 k+1}\right), \\
d\left(x_{2 k}, x_{2 k+1}\right), \\
\frac{d\left(x_{2 k+1}, x_{2 k}\right)+d\left(x_{2 k+2}, x_{2 k+1}\right)}{2}, \\
\frac{d\left(x_{2 k+1}, x_{2 k}\right)+d\left(x_{2 k}, x_{2 k+1}\right)}{2}, \\
\frac{d\left(x_{2 k+2}, x_{2 k+1}\right)+d\left(x_{2 k}, x_{2 k+1}\right)}{2}, \\
\frac{d\left(x_{2 k+1}, x_{2 k+1}\right)+d\left(x_{2 k+2}, x_{2 k}\right)}{2}
\end{array}\right) .
\end{aligned}
$$

Suppose that $d\left(x_{2 k+1}, x_{2 k+2}\right)>d\left(x_{2 k}, x_{2 k+1}\right)=0$. It follows from the definition of $\phi$ that

$$
\begin{aligned}
& s^{4} d\left(x_{2 k+1}, x_{2 k+2}\right) \\
& \leq \phi\left(\begin{array}{l}
d\left(x_{2 k+1}, x_{2 k+2}\right), d\left(x_{2 k+1}, x_{2 k+2}\right), \\
d\left(x_{2 k+1}, x_{2 k+2}\right), \\
d\left(x_{2 k+1}, x_{2 k+2}\right), d\left(x_{2 k+1}, x_{2 k+2}\right), \\
d\left(x_{2 k+1}, x_{2 k+2}\right), s d\left(x_{2 k+1}, x_{2 k+2}\right)
\end{array}\right) \\
& \leq \phi\left(\begin{array}{l}
s^{4} d\left(x_{2 k+1}, x_{2 k+2}\right), s^{4} d\left(x_{2 k+1}, x_{2 k+2}\right), \\
s^{4} d\left(x_{2 k+1}, x_{2 k+2}\right), \\
s^{4} d\left(x_{2 k+1}, x_{2 k+2}\right), s^{4} d\left(x_{2 k+1}, x_{2 k+2}\right), \\
s^{4} d\left(x_{2 k+1}, x_{2 k+2}\right), s^{4} d\left(x_{2 k+1}, x_{2 k+2}\right)
\end{array}\right) \\
& =\bar{\phi}\left(s^{4} d\left(x_{2 k+1}, x_{2 k+2}\right)\right)<s^{4} d\left(x_{2 k+1}, x_{2 k+2}\right),
\end{aligned}
$$

which is a contradiction. Therefore, $d\left(x_{2 k+1}, x_{2 k+2}\right)=0$. By the definition of the sequence $\left\{x_{n}\right\}$, it means that $x_{2 k}=A x_{2 k}=B x_{2 k}$. That is, $x_{2 k}$ is a common fixed point of $A$ and $B$.

If $n=2 k+1$, then using the same arguments in the case $x_{2 k}=x_{2 k+1}$, it can be shown that $x_{2 k+1}$ is a common fixed point of $A$ and $B$.

From now on, we suppose that $x_{n} \neq x_{n+1}$ for all $n \in N_{0}$. Now we shall prove that

$s^{4} d\left(x_{n}, x_{n+1}\right) \leq \bar{\phi}\left(s^{4} d\left(x_{n}, x_{n+1}\right)\right)$, for each $n \in N_{0}$. (2)

We consider two cases:

Case I: $n=2 k, k \in N$. From the contraction condition (1) with $x=x_{2 k}$ and $y=x_{2 k-1}$, we get

$$
\begin{aligned}
& s^{4} d\left(x_{2 k+1}, x_{2 k}\right)=s^{4} d\left(A x_{2 k}, B x_{2 k-1}\right) \\
& d\left(A x_{2 k}, x_{2 k}\right), d\left(B x_{2 k-1}, x_{2 k-1}\right), \\
& d\left(x_{2 k}, x_{2 k-1}\right), \frac{d\left(A x_{2 k}, x_{2 k}\right)+d\left(B x_{2 k-1}, x_{2 k-1}\right)}{2}, \\
& \leq \phi\left(\begin{array}{l}
\frac{d\left(A x_{2 k}, x_{2 k}\right)+d\left(x_{2 k}, x_{2 k-1}\right)}{2}, \\
\frac{d\left(B x_{2 k-1}, x_{2 k-1}\right)+d\left(x_{2 k}, x_{2 k-1}\right)}{2}, \\
\left.\frac{d\left(A x_{2 k}, x_{2 k-1}\right)+d\left(B x_{2 k-1}, x_{2 k}\right)}{2}\right) \\
\frac{d\left(x_{2 k+1}, x_{2 k}\right), d\left(x_{2 k}, x_{2 k-1}\right), d\left(x_{2 k}, x_{2 k-1}\right),}{2}, \\
\frac{d\left(x_{2 k+1}, x_{2 k}\right)+d\left(x_{2 k}, x_{2 k-1}\right)}{2}, \\
\frac{d\left(x_{2 k+1}, x_{2 k}\right)+d\left(x_{2 k}, x_{2 k-1}\right)}{2}, \\
\frac{d\left(x_{2 k}, x_{2 k-1}\right)+d\left(x_{2 k}, x_{2 k-1}\right)}{2}, \\
\frac{d\left(x_{2 k+1}, x_{2 k-1}\right)+d\left(x_{2 k}, x_{2 k}\right)}{2}
\end{array}\right)
\end{aligned}
$$

If $d\left(x_{2 k+1}, x_{2 k}\right)>d\left(x_{2 k}, x_{2 k-1}\right)$, by virtue of the definition of $\phi$, one can obtain 


$$
\begin{aligned}
& s^{4} d\left(x_{2 k+1}, x_{2 k}\right) \\
& \leq \phi\left(\begin{array}{l}
d\left(x_{2 k+1}, x_{2 k}\right), d\left(x_{2 k+1}, x_{2 k}\right), d\left(x_{2 k+1}, x_{2 k}\right), \\
d\left(x_{2 k+1}, x_{2 k}\right), d\left(x_{2 k+1}, x_{2 k}\right), \\
d\left(x_{2 k+1}, x_{2 k}\right), s d\left(x_{2 k+1}, x_{2 k}\right)
\end{array}\right) \\
& \leq \phi\left(\begin{array}{l}
s^{4} d\left(x_{2 k+1}, x_{2 k}\right), s^{4} d\left(x_{2 k+1}, x_{2 k}\right), \\
s^{4} d\left(x_{2 k+1}, x_{2 k}\right), \\
s^{4} d\left(x_{2 k+1}, x_{2 k}\right), s^{4} d\left(x_{2 k+1}, x_{2 k}\right), \\
s^{4} d\left(x_{2 k+1}, x_{2 k}\right), s^{4} d\left(x_{2 k+1}, x_{2 k}\right)
\end{array}\right) \\
& =\bar{\phi}\left(s^{4} d\left(x_{2 k+1}, x_{2 k}\right)\right)<s^{4} d\left(x_{2 k+1}, x_{2 k}\right),
\end{aligned}
$$

a contradiction. It follows that

$$
d\left(x_{2 k+1}, x_{2 k}\right) \leq d\left(x_{2 k}, x_{2 k-1}\right) .
$$

Hence,

$$
s^{4} d\left(x_{2 k+1}, x_{2 k}\right) \leq \bar{\phi}\left(s^{4} d\left(x_{2 k}, x_{2 k-1}\right)\right) \text {, for each } k \in N . \text { (3) }
$$

Case II: $n=2 k+1, k \in N_{0}$. Using the same technique in proving the case I, it can be proved that (2) holds for $n=2 k+1$. That is,

$$
\begin{aligned}
& s^{4} d\left(x_{2 k+1}, x_{2 k+2}\right) \leq \bar{\phi}\left(s^{4} d\left(x_{2 k+1}, x_{2 k}\right)\right), \\
& \text { for each } k \in N_{0} .
\end{aligned}
$$

From (3) and (4), we conclude that (2) holds for all $n \in N_{0}$.

Since $\bar{\phi}(t)<t$ for all $t>0$, using Lemma 2.3, we obtain that $\lim _{n \rightarrow+\infty} \bar{\phi}^{n}(t)=0$ for all $t>0$. It follows that

$$
\lim _{n \rightarrow+\infty} d\left(x_{n}, x_{n+1}\right)=0
$$

Now we prove that $\left\{x_{n}\right\}$ is a Cauchy sequence. To do this, it is sufficient to show that the subsequence $\left\{x_{2 n}\right\}$ is a Cauchy sequence in $X$. Assume on the contrary that $\left\{x_{2 n}\right\}$ is not a Cauchy sequence. Then there exists $\varepsilon>0$ for which we can find subsequence $\left\{x_{2 m_{k}}\right\}$ and $\left\{x_{2 n_{k}}\right\}$ so that $2 m_{k}$ is the smallest index for which $2 m_{k}>2 n_{k}>k$,

$$
d\left(x_{2 m_{k}}, x_{2 n_{k}}\right) \geq \varepsilon
$$

and

$$
d\left(x_{2 m_{k}-2}, x_{2 n_{k}}\right)<\varepsilon .
$$

Using the triangle inequality in $b$-metric space and (6), we have

$$
\begin{aligned}
\varepsilon & \leq d\left(x_{2 m_{k}}, x_{2 n_{k}}\right) \\
& \leq \operatorname{sd}\left(x_{2 m_{k}-2}, x_{2 n_{k}}\right)+s^{2} d\left(x_{2 m_{k}-2}, x_{2 m_{k}-1}\right) \\
& +s^{2} d\left(x_{2 m_{k}-1}, x_{2 m_{k}}\right) \\
\leq & \varepsilon s+s^{2} d\left(x_{2 m_{k}-2}, x_{2 m_{k}-1}\right)+s^{2} d\left(x_{2 m_{k}-1}, x_{2 m_{k}}\right) .
\end{aligned}
$$

Taking the upper limit as $k \rightarrow+\infty$, one can obtain

$$
\varepsilon \leq \limsup _{k \rightarrow+\infty} d\left(x_{2 m_{k}}, x_{2 n_{k}}\right) \leq \varepsilon s .
$$

Also,

$$
\begin{aligned}
& \varepsilon \leq \limsup _{k \rightarrow+\infty} d\left(x_{2 m_{k}}, x_{2 n_{k}}\right) \\
& \leq \operatorname{sd}\left(x_{2 m_{k}}, x_{2 m_{k}-1}\right)+\operatorname{sd}\left(x_{2 m_{k}-1}, x_{2 n_{k}}\right),
\end{aligned}
$$

hence,

$$
\frac{\varepsilon}{s} \leq \limsup _{k \rightarrow+\infty} d\left(x_{2 m_{k}-1}, x_{2 n_{k}}\right) .
$$

On the other hand, we get

$d\left(x_{2 m_{k}-1}, x_{2 n_{k}}\right) \leq s d\left(x_{2 m_{k}-1}, x_{2 m_{k}}\right)+s d\left(x_{2 m_{k}}, x_{2 n_{k}}\right)$.

It follows from (5) and (8) that

$\limsup _{k \rightarrow+\infty} d\left(x_{2 m_{k}-1}, x_{2 n_{k}}\right) \leq s \limsup _{k \rightarrow+\infty} d\left(x_{2 m_{k}}, x_{2 n_{k}}\right) \leq s^{2} \varepsilon$.

Consequently,

$$
\frac{\varepsilon}{s} \leq \limsup _{k \rightarrow+\infty} d\left(x_{2 m_{k}-1}, x_{2 n_{k}}\right) \leq s^{2} \varepsilon .
$$

Similarly, we deduce that

$$
\frac{\varepsilon}{s^{2}} \leq \limsup _{k \rightarrow+\infty} d\left(x_{2 m_{k}-1}, x_{2 n_{k}+1}\right) \leq s^{3} \varepsilon \text {. }
$$

Using the triangle inequality in $b$-metric space and contraction condition (1), we have

$$
\begin{aligned}
& d\left(x_{2 n_{k}}, x_{2 m_{k}}\right) \\
& \leq \operatorname{sd}\left(x_{2 n_{k}}, x_{2 n_{k}+1}\right)+\operatorname{sd}\left(x_{2 n_{k}+1}, x_{2 m_{k}}\right) \\
& \leq \operatorname{sd}\left(x_{2 n_{k}}, x_{2 n_{k}+1}\right)+\operatorname{sd}\left(A x_{2 n_{k}}, B x_{2 m_{k}-1}\right) \\
& \leq \operatorname{sd}\left(x_{2 n_{k}}, x_{2 n_{k}+1}\right) \\
& +\frac{1}{s^{3}} \phi\left(\begin{array}{l}
\frac{d\left(A x_{2 n_{k}}, x_{2 n_{k}}\right), d\left(B x_{2 m_{k}-1}, x_{2 m_{k}-1}\right), d\left(x_{2 n_{k}}, x_{2 m_{k}-1}\right),}{2}, \\
\left.\frac{d\left(A x_{2 n_{k}}, x_{2 n_{k}}\right)+d\left(B x_{2 m_{k}-1}, x_{2 m_{k}-1}\right)}{2}, x_{2 n_{k}}\right)+d\left(x_{2 n_{k}}, x_{2 m_{k}-1}\right) \\
\frac{d\left(B x_{2 m_{k}-1}, x_{2 m_{k}-1}\right)+d\left(x_{2 n_{k}}, x_{2 m_{k}-1}\right)}{2}, \\
\frac{d\left(A x_{2 n_{k}}, x_{2 m_{k}-1}\right)+d\left(B x_{2 m_{k}-1}, x_{2 n_{k}}\right)}{2}
\end{array}\right) \\
& =\operatorname{sd}\left(x_{\left.2 n_{k}, x_{2 n_{k}+1}\right)}\right)
\end{aligned}
$$

$$
+\frac{1}{s^{3}} \phi\left(\begin{array}{l}
\frac{d\left(x_{2 n_{k}+1}, x_{2 n_{k}}\right), d\left(x_{2 m_{k}}, x_{2 m_{k}-1}\right), d\left(x_{2 n_{k}}, x_{2 m_{k}-1}\right)}{\frac{d\left(x_{2 n_{k}+1}, x_{2 n_{k}}\right)+d\left(x_{2 m_{k}}, x_{2 m_{k}-1}\right)}{2}} \\
\frac{d\left(x_{2 n_{k}+1}, x_{2 n_{k}}\right)+d\left(x_{2 n_{k}}, x_{2 m_{k}-1}\right)}{2}, \\
\frac{d\left(x_{2 m_{k}+1}, x_{2 m_{k}-1}\right)+d\left(x_{2 n_{k}}, x_{2 m_{k}-1}\right)}{2},
\end{array}\right)
$$


In view of above inequality and (5), (9), (10), one can obtain that

$$
\begin{aligned}
& \varepsilon \leq \limsup _{k \rightarrow+\infty} d\left(x_{2 n_{k}}, x_{2 m_{k}}\right) \\
& \leq 0+\frac{1}{s^{3}} \phi\left(0,0, \varepsilon s^{2}, 0, \varepsilon s^{2}, \frac{\varepsilon s^{2}}{2}, \frac{\varepsilon s^{3}+\varepsilon s}{2}\right) \\
& \leq \frac{1}{s^{3}} \phi\left(\varepsilon s^{3}, \varepsilon s^{3}, \varepsilon s^{3}, \varepsilon s^{3}, \varepsilon s^{3}, \varepsilon s^{3}, \varepsilon s^{3}\right) \\
& =\frac{1}{s^{3}} \bar{\phi}\left(\varepsilon s^{3}\right) \\
& <\varepsilon .
\end{aligned}
$$

It is a contradiction and it follows that $\left\{x_{2 n}\right\}$ is a Cauchy sequence in $X$. Since $X$ is complete, there exists $x^{*}$ such that

$$
\begin{aligned}
& \lim _{n \rightarrow+\infty} x_{2 n+1}=\lim _{n \rightarrow+\infty} A x_{2 n} \\
& =\lim _{n \rightarrow+\infty} x_{2 n+2}=\lim _{n \rightarrow+\infty} B x_{2 n+1}=x^{*} .
\end{aligned}
$$

Without loss of generality, we suppose $A$ is continuous. It follows that

$$
x^{*}=\lim _{n \rightarrow+\infty} x_{2 n+1}=\lim _{n \rightarrow+\infty} A x_{2 n}=A \lim _{n \rightarrow+\infty} x_{2 n}=A x^{*} .
$$

This implies that $x^{*}$ is a fixed point of $A$.

Next, we show that $x^{*}$ is a fixed point of \$B.\$ In view of the contraction condition (1), we get that

$$
\begin{aligned}
& s^{4} d\left(B x^{*}, x^{*}\right) \\
& =s^{4} d\left(B x^{*}, A x^{*}\right) \\
& \leq \phi\left(\begin{array}{l}
\frac{d\left(A x^{*}, x^{*}\right), d\left(B x^{*}, x^{*}\right), d\left(x^{*}, x^{*}\right),}{\frac{d\left(A x^{*}, x^{*}\right)+d\left(B x^{*}, x^{*}\right)}{2},} \\
\frac{d\left(B x^{*}, x^{*}\right)+d\left(x^{*}, x^{*}\right)}{2}, \\
\left.\frac{d\left(A x^{*}, x^{*}\right)+d\left(B x^{*}, x^{*}\right)}{2}, x^{*}\right)
\end{array}\right), \\
& =\phi\left(0, d\left(B x^{*}, x^{*}\right), 0, \frac{d\left(B x^{*}, x^{*}\right)}{2}, 0, \frac{d\left(B x^{*}, x^{*}\right)}{2}, \frac{d\left(B x^{*}, x^{*}\right)}{2}\right) .
\end{aligned}
$$

If suppose that $d\left(B x^{*}, x^{*}\right)>0$, then we have

$$
\begin{aligned}
& s^{4} d\left(B x^{*}, x^{*}\right) \\
& \leq \phi\left(\begin{array}{l}
d\left(B x^{*}, x^{*}\right), d\left(B x^{*}, x^{*}\right), \\
d\left(B x^{*}, x^{*}\right), d\left(B x^{*}, x^{*}\right), \\
d\left(B x^{*}, x^{*}\right), d\left(B x^{*}, x^{*}\right), d\left(B x^{*}, x^{*}\right)
\end{array}\right) \\
& =\bar{\phi}\left(d\left(B x^{*}, x^{*}\right)\right)<d\left(B x^{*}, x^{*}\right),
\end{aligned}
$$

a contradiction. It follows that $d\left(B x^{*}, x^{*}\right)=0$. That is, $x^{*}$ is also a fixed point of $B$.

Assume that $y^{*}$ is another common fixed point of $A$ and $B$, that is, $d\left(x^{*}, y^{*}\right)>0$. Then

$$
\begin{aligned}
& s^{4} d\left(x^{*}, y^{*}\right) \\
& =s^{4} d\left(A x^{*}, B y^{*}\right) \\
& \leq\left(\begin{array}{l}
d\left(A x^{*}, x^{*}\right), d\left(B y^{*}, y^{*}\right), d\left(x^{*}, y^{*}\right), \\
\frac{d\left(A x^{*}, x^{*}\right)+d\left(B y^{*}, y^{*}\right)}{2}, \\
\frac{d\left(A x^{*}, x^{*}\right)+d\left(x^{*}, y^{*}\right)}{2}, \\
\frac{d\left(B y^{*}, y^{*}\right)+d\left(x^{*}, y^{*}\right)}{2},
\end{array}\right), \\
& =\phi\left(0,0, d\left(x^{*}, y^{*}\right), 0, \frac{d\left(x^{*}, y^{*}\right)+d\left(B y^{*}, x^{*}\right)}{2}, \frac{d\left(x^{*}, y^{*}\right)}{2}, d\left(x^{*}, y^{*}\right)\right) \\
& \leq \bar{\phi}\left(d\left(x^{*}, y^{*}\right)\right)<d\left(x^{*}, y^{*}\right),
\end{aligned}
$$

which is a contradiction. It follows that $x^{*}$ is a unique common fixed point in $X$. This completes the proof.

If $A=B$ in Theorem 1 , then we get that:

Corollary 3.2. Let $(X, d)$ be a complete $b$-metric space with constant $s \geq 1$ and $A: X \rightarrow X$ be a continuous mapping. If there exists $\phi \in \Phi$ such that

$$
\begin{aligned}
& s^{4} d(A x, A y) \\
& \leq \phi\left(\begin{array}{l}
d(A x, x), d(A y, y), d(x, y), \\
\frac{d(A x, x)+d(A y, y)}{2}, \frac{d(A x, x)+d(x, y)}{2}, \\
\left.\frac{d(A y, y)+d(x, y)}{2}, \frac{d(A x, y)+d(A y, x)}{2}\right)
\end{array}\right)
\end{aligned}
$$

for all $x, y \in X$, then $A$ has a unique fixed point $x^{*} \in X$.

Theorem 3.3. Let $(X, d)$ be a complete $b$-metric space with constant $s \geq 1$. Suppose $A$ and $B: X \rightarrow X$ are two mappings and one of them is continuous. If there exists $\psi \in \Psi$ such that

$$
\begin{aligned}
& s^{6} d^{2}(A x, B y) \\
& \leq \psi\left(\begin{array}{l}
d(A x, x) d(B y, y), d(A x, x) d(x, y), \\
d(B y, y) d(x, y), d(A x, B y) d(x, y), \\
d(A x, B y) d(A x, x), d(A x, B y) d(B y, y), \\
\left.d^{2}(A x, x), d^{2}(B y, y), d^{2}(x, y)\right)
\end{array}\right)
\end{aligned}
$$

for all $x, y \in X$, then $A$ and $B$ have a unique common fixed point $x^{*} \in X$. 
Proof. Let $x_{0} \in X$ be arbitrary. We define a sequence $\left\{x_{n}\right\}$ as follows:

$$
x_{2 n+1}=A x_{2 n}, x_{2 n+2}=B x_{2 n+1}, n \in N_{0} .
$$

We now suppose that $d\left(x_{n}, x_{n+1}\right)>0$ for every \$n.\$ Otherwise, there exists some $n \in N_{0}$ such that $x_{n}=x_{n+1}$. If $n=2 k$, from the contraction condition (11) with $x=x_{2 k}$ and $y=x_{2 k+1}$, one can obtain

$$
\begin{aligned}
& s^{6} d^{2}\left(x_{2 k+1}, x_{2 k+2}\right) \\
& =s^{6} d^{2}\left(A x_{2 k}, B x_{2 k+1}\right) \\
& \leq \psi\left(\begin{array}{l}
d\left(A x_{2 k}, x_{2 k}\right) d\left(B x_{2 k+1}, x_{2 k+1}\right), \\
d\left(A x_{2 k}, x_{2 k}\right) d\left(x_{2 k}, x_{2 k+1}\right), \\
d\left(B x_{2 k+1}, x_{2 k+1}\right) d\left(x_{2 k}, x_{2 k+1}\right), \\
d\left(A x_{2 k}, B x_{2 k+1}\right) d\left(x_{2 k}, x_{2 k+1}\right), \\
d\left(A x_{2 k}, B x_{2 k+1}\right) d\left(A x_{2 k}, x_{2 k}\right), \\
d\left(A x_{2 k}, B x_{2 k+1}\right) d\left(B x_{2 k+1}, x_{2 k+1}\right), \\
d^{2}\left(A x_{2 k}, x_{2 k}\right), d^{2}\left(B x_{2 k+1}, x_{2 k+1}\right), d^{2}\left(x_{2 k}, x_{2 k+1}\right)
\end{array}\right) \\
& =\psi\left(\begin{array}{l}
d\left(x_{2 k+1}, x_{2 k}\right) d\left(x_{2 k+2}, x_{2 k+1}\right), \\
d\left(x_{2 k+1}, x_{2 k}\right) d\left(x_{2 k}, x_{2 k+1}\right), \\
d\left(x_{2 k+2}, x_{2 k+1}\right) d\left(x_{2 k}, x_{2 k+1}\right), \\
d\left(x_{2 k+1}, x_{2 k+2}\right) d\left(x_{2 k}, x_{2 k+1}\right), \\
d\left(x_{2 k+1}, x_{2 k+2}\right) d\left(x_{2 k+1}, x_{2 k}\right), \\
d\left(x_{2 k+1}, x_{2 k+2}\right) d\left(x_{2 k+2}, x_{2 k+1}\right), \\
d^{2}\left(x_{2 k+1}, x_{2 k}\right), d^{2}\left(x_{2 k+2}, x_{2 k+1}\right), \\
\left.d^{2}\left(x_{2 k}, x_{2 k+1}\right)\right) .
\end{array}\right) .
\end{aligned}
$$

We suppose that $d\left(x_{2 k+1}, x_{2 k+2}\right)>d\left(x_{2 k}, x_{2 k+1}\right)=0$. By the definition of $\psi$, we have

$$
\begin{aligned}
& s^{6} d^{2}\left(x_{2 k+1}, x_{2 k+2}\right) \\
& \leq \psi\left(0,0,0,0,0, d^{2}\left(x_{2 k+1}, x_{2 k+2}\right), 0, d^{2}\left(x_{2 k+1}, x_{2 k+2}\right), 0\right) \\
& \leq \psi\left(\begin{array}{l}
d^{2}\left(x_{2 k+1}, x_{2 k+2}\right), d^{2}\left(x_{2 k+1}, x_{2 k+2}\right), \\
d^{2}\left(x_{2 k+1}, x_{2 k+2}\right), d^{2}\left(x_{2 k+1}, x_{2 k+2}\right), \\
d^{2}\left(x_{2 k+1}, x_{2 k+2}\right), d^{2}\left(x_{2 k+1}, x_{2 k+2}\right), \\
d^{2}\left(x_{2 k+1}, x_{2 k+2}\right), d^{2}\left(x_{2 k+1}, x_{2 k+2}\right), \\
d^{2}\left(x_{2 k+1}, x_{2 k+2}\right)
\end{array}\right) \\
& =\bar{\psi}\left(d^{2}\left(x_{2 k+1}, x_{2 k+2}\right)\right)<d^{2}\left(x_{2 k+1}, x_{2 k+2}\right),
\end{aligned}
$$

a contradiction. Hence, $d\left(x_{2 k+1}, x_{2 k+2}\right)=0$. It follows from the definition of the sequence $\left\{x_{n}\right\}$ that

$$
x_{2 k}=A x_{2 k}=B x_{2 k} \text {. }
$$

That is, $x_{2 k}$ is a common fixed point of $A$ and $B$.

Similarly, if $n=2 k+1$, we can prove that $x_{2 k+1}$ is a common fixed point of $A$ and $B$.
From now on, we suppose that $x_{n} \neq x_{n+1}$ for all $n \in N_{0}$. Using the similar argument in the proof of Theorem 3.1, one can deduce that

$$
d^{2}\left(x_{n}, x_{n+1}\right) \leq \bar{\psi}\left(d^{2}\left(x_{n}, x_{n-1}\right)\right) \text {, for each } n \in N .
$$

It follows from Lemma 2.3 that $\lim \bar{\psi}^{n}(t)=0$ for all $t>0$, which implies that

$$
\mathrm{S} \lim _{n \rightarrow+\infty} d\left(x_{n}, x_{n+1}\right)=0 .
$$

Next we prove that $\left\{x_{n}\right\}$ is a Cauchy sequence. Obviously, it is sufficient to show that the subsequence $\left\{x_{2 n}\right\}$ is a Cauchy sequence in $X$. As in the proof of Theorem 3.1, we obtain that inequalities (9),(10) hold, and

$$
\frac{\varepsilon}{s} \leq \limsup _{k \rightarrow+\infty} d\left(x_{2 m_{k}}, x_{2 n_{k}+1}\right) \leq s^{2} \varepsilon .
$$

The triangle inequality in $b$-metric space and contraction condition (11) ensure that

$$
\begin{aligned}
& d^{2}\left(x_{2 n_{k}}, x_{2 m_{k}}\right) \\
& \leq\left(s d\left(x_{2 n_{k}}, x_{2 n_{k}+1}\right)+s d\left(x_{2 n_{k}+1}, x_{2 m_{k}}\right)^{2}\right. \\
& =s^{2} d\left(x_{2 n_{k}}, x_{2 n_{k}+1}\right)+2 s^{2} d\left(x_{2 n_{k}}, x_{2 n_{k}+1}\right) d\left(x_{2 n_{k}+1}, x_{2 m_{k}}\right) \\
& +s^{2} d\left(A x_{2 n_{k}}, B x_{2 m_{k}-1}\right) \\
& \leq s^{2} d\left(x_{2 n_{k}}, x_{2 n_{k}+1}\right) \\
& +2 s^{2} d\left(x_{2 n_{k}}, x_{2 n_{k}+1}\right) d\left(x_{2 n_{k}+1}, x_{2 m_{k}}\right) \\
& \left(d\left(A x_{2 n_{k}}, x_{2 n_{k}}\right) d\left(B x_{2 m_{k}-1}, x_{2 m_{k}-1}\right),\right. \\
& d\left(A x_{2 n_{k}}, x_{2 n_{k}}\right) d\left(x_{2 n_{k}}, x_{2 m_{k}-1}\right) \text {, } \\
& d\left(B x_{2 m_{k}-1}, x_{2 m_{k}-1}\right) d\left(x_{2 n_{k}}, x_{2 m_{k}-1}\right) \text {, } \\
& +\frac{1}{s} \psi \mid \begin{array}{l}
d\left(A x_{2 n_{k}}, B x_{2 m_{k}-1}\right) d\left(x_{2 n_{k}}, x_{2 m_{k}-1}\right), \\
d\left(A x_{2 n_{k}}, B x_{2 m_{k}-1}\right) d\left(A x_{2 n_{k}}, x_{2 n_{k}}\right),
\end{array} \\
& d\left(A x_{2 n_{k}}, B x_{2 m_{k}-1}\right) d\left(B x_{2 m_{k}-1}, x_{2 m_{k}-1}\right) \text {, } \\
& d^{2}\left(A x_{2 n_{k}}, x_{2 n_{k}}\right), d^{2}\left(B x_{2 m_{k}-1}, x_{2 m_{k}-1}\right) \text {, } \\
& \left.d^{2}\left(x_{2 n_{k}}, x_{2 m_{k}-1}\right)\right) \\
& \leq s^{2} d\left(x_{2 n_{k}}, x_{2 n_{k}+1}\right) \\
& \left.+2 s^{2} d\left(x_{2 n_{k}}, x_{2 n_{k}+1}\right) d\left(x_{2 n_{k}+1}, x_{2 m_{k}}\right)\right) \\
& +\frac{1}{s^{4}} \psi\left(\begin{array}{l}
d\left(x_{2 n_{k}+1}, x_{2 n_{k}}\right) d\left(x_{2 m_{k}}, x_{2 m_{k}-1}\right), \\
d\left(x_{2 n_{k}+1}, x_{2 n_{k}}\right) d\left(x_{2 n_{k}}, x_{2 m_{k}-1}\right), \\
d\left(x_{2 m_{k}}, x_{2 m_{k}-1}\right) d\left(x_{2 n_{k}}, x_{2 m_{k}-1}\right), \\
d\left(x_{2 n_{k}+1}, x_{2 m_{k}}\right) d\left(x_{2 n_{k}}, x_{2 m_{k}-1}\right), \\
d\left(x_{2 n_{k}+1}, x_{2 m_{k}}\right) d\left(x_{2 n_{k}+1}, x_{2 n_{k}}\right),
\end{array}\right. \\
& d\left(x_{2 n_{k}+1}, x_{2 m_{k}}\right) d\left(x_{2 m_{k}}, x_{2 m_{k}-1}\right) \text {, } \\
& d^{2}\left(x_{2 n_{k}+1}, x_{2 n_{k}}\right), d^{2}\left(x_{2 m_{k}}, x_{2 m_{k}+1}\right), \\
& \left.d^{2}\left(x_{2 n_{k}}, x_{2 m_{k}-1}\right)\right) \text {. }
\end{aligned}
$$


In light of above inequality and (9), (10), (13) and (14), we have

$$
\begin{aligned}
& \varepsilon^{2} \leq \limsup _{k \rightarrow+\infty} d^{2}\left(x_{2 m_{k}}, x_{2 n_{k}}\right) \\
& \leq 0+0+\frac{1}{s^{4}} \psi\left(0,0,0, \varepsilon^{2} s^{4}, 0,0,0,0, \varepsilon^{2} s^{4}\right) \\
& \leq \frac{1}{s^{4}} \psi\left(\begin{array}{l}
\varepsilon^{2} s^{4}, \varepsilon^{2} s^{4}, \varepsilon^{2} s^{4}, \varepsilon^{2} s^{4}, \\
\varepsilon^{2} s^{4}, \varepsilon^{2} s^{4}, \varepsilon^{2} s^{4}, \varepsilon^{2} s^{4}, \varepsilon^{2} s^{4}
\end{array}\right) \\
& =\frac{1}{s^{4}} \bar{\psi}\left(\varepsilon^{2} s^{4}\right) \\
& <\varepsilon^{2} .
\end{aligned}
$$

It is a contradiction. Hence, $\left\{x_{2 n}\right\}$ is a Cauchy sequence in $X$. The completeness of $X$ ensures that there exists $x^{*}$ such that

$\lim _{n \rightarrow+\infty} x_{2 n+1}=\lim _{n \rightarrow+\infty} A x_{2 n}=\lim _{n \rightarrow+\infty} x_{2 n+2}=\lim _{n \rightarrow+\infty} B x_{2 n+1}=x^{*}$.

Without loss of generality, we suppose $A$ is continuous. It follows that

$$
x^{*}=\lim _{n \rightarrow+\infty} x_{2 n+1}=\lim _{n \rightarrow+\infty} A x_{2 n}=A \lim _{n \rightarrow+\infty} x_{2 n}=A x^{*} .
$$

That is, $x^{*}$ is a fixed point of $A$.

Next, we shall prove that $x^{*}$ is a fixed point of $B$. By the contraction condition (11), we obtain that

$$
\begin{aligned}
& s^{6} d^{2}\left(x^{*}, B x^{*}\right)=s^{6} d^{2}\left(A x^{*}, B x^{*}\right) \\
& \leq \psi\left(\begin{array}{l}
d\left(A x^{*}, x^{*}\right) d\left(B x^{*}, x^{*}\right), d\left(A x^{*}, x^{*}\right) d\left(x^{*}, x^{*}\right), \\
d\left(B x^{*}, x^{*}\right) d\left(x^{*}, x^{*}\right), d\left(A x^{*}, B x^{*}\right) d\left(x^{*}, x^{*}\right), \\
d\left(A x^{*}, B x^{*}\right) d\left(A x^{*}, x^{*}\right), \\
d\left(A x^{*}, B x^{*}\right) d\left(B x^{*}, x^{*}\right), \\
d^{2}\left(A x^{*}, x^{*}\right), d^{2}\left(B x^{*}, x^{*}\right), d^{2}\left(x^{*}, x^{*}\right)
\end{array}\right) \\
& =\psi\left(0,0,0,0,0, d^{2}\left(B x^{*}, x^{*}\right), 0, d^{2}\left(B x^{*}, x^{*}\right), 0\right) .
\end{aligned}
$$

If we suppose that $d\left(B x^{*}, x^{*}\right)>0$, then one can get

$$
s^{6} d^{2}\left(B x^{*}, x^{*}\right) \leq \bar{\psi}\left(d^{2}\left(B x^{*}, x^{*}\right)\right)<d^{2}\left(B x^{*}, x^{*}\right),
$$

which is a contradiction. Hence, we deduce that $x^{*}$ is also a fixed point of $B$.

Suppose that $x^{*}$ and $y^{*}$ are different common fixed points of $A$ and $B$, then we obtain that

$$
\begin{aligned}
& s^{6} d^{2}\left(x^{*}, y^{*}\right)=s^{6} d^{2}\left(A x^{*}, B y^{*}\right) \\
& \leq \psi\left(\begin{array}{l}
d\left(A x^{*}, x^{*}\right) d\left(B y^{*}, y^{*}\right), d\left(A x^{*}, x^{*}\right) d\left(x^{*}, y^{*}\right), \\
d\left(B y^{*}, y^{*}\right) d\left(x^{*}, y^{*}\right), d\left(A x^{*}, B y^{*}\right) d\left(x^{*}, y^{*}\right), \\
d\left(A x^{*}, B y^{*}\right) d\left(A x^{*}, x^{*}\right), d\left(A x^{*}, B y^{*}\right) d\left(B y^{*}, y^{*}\right), \\
\left.d^{2}\left(A x^{*}, x^{*}\right), d^{2}\left(B y^{*}, y^{*}\right), d^{2}\left(x^{*}, y^{*}\right)\right)
\end{array}\right) \\
& =\psi\left(0,0,0, d^{2}\left(x^{*}, y^{*}\right), 0,0,0,0, d^{2}\left(x^{*}, y^{*}\right)\right) \\
& \leq \bar{\psi}\left(d^{2}\left(x^{*}, y^{*}\right)\right)<d^{2}\left(x^{*}, y^{*}\right),
\end{aligned}
$$

a contradiction. Consequently, $x^{*}$ is a unique common fixed point in $X$. This completes the proof.

If $A=B$ in Theorem 3 , we have the following result.

Corollary 3.4. Let $(X, d)$ be a complete $b$-metric space with constant $s \geq 1$. Suppose $A: X \rightarrow X$ be a continuous mapping. If there exists $\psi \in \Psi$ such that

$$
\begin{aligned}
& s^{6} d^{2}(A x, A y) \\
& \leq \psi\left(\begin{array}{l}
d(A x, x) d(A y, y), d(A x, x) d(x, y), \\
d(A y, y) d(x, y), d(A x, A y) d(x, y), \\
d(A x, A y) d(A x, x), d(A x, A y) d(A y, y), \\
d^{2}(A x, x), d^{2}(A y, y), d^{2}(x, y)
\end{array}\right)
\end{aligned}
$$

for all $x, y \in X$, then $A$ has a unique common fixed point $x^{*} \in X$.

\section{Examples}

Example 4.1. Let $X=[0,1]$ endowed with the $b$-metric:

$$
d: X \times X \rightarrow[0,+\infty), d(x, y)=|x-y|^{2}
$$

with constant $s=2$. Consider mappings $A, B: X \times X$ by $A x=\frac{x}{16}$ and $B x=\frac{x}{32}$. Define the mapping $\phi:\left(\mathbb{R}^{+}\right)^{7} \rightarrow \mathbb{R}^{+}$by

$$
\phi\left(x_{1}, x_{2}, x_{3}, x_{4}, x_{5}, x_{6}, x_{7}\right)=\frac{1}{7}\left(\sum_{i=1}^{7} \frac{x_{i}}{1+x_{i}}\right) .
$$

Clearly, $(X, d)$ is a complete $b$-metric space and $A$ is continuous with respect to $d$. So we verify the contraction condition (1).

By calculus, we have

$$
\begin{aligned}
& s^{4} d(A x, B y)=16\left|\frac{x}{16}-\frac{y}{32}\right|^{2} \\
& \leq \frac{x^{2}}{16}+\frac{y^{2}}{64}<\frac{1}{7}\left(\frac{225 x^{2}}{512}+\frac{961 y^{2}}{2048}\right) \\
& \leq \frac{1}{7}\left(\frac{d(A x, x)}{1+d(A x, x)}+\frac{d(B y, y)}{1+d(B y, y)}\right) \\
& \leq \phi\left(\begin{array}{l}
\left.\frac{d(A x, x), d(B y, y), d(x, y), \frac{d(A x, x)+d(B y, y)}{2},}{2}, x\right)+d(x, y) \\
\frac{d(A x, y)+d(B y, x)}{2}
\end{array}\right) .
\end{aligned}
$$

Therefore, we show that the contraction condition (1) is satisfied. It follows that we can apply Theorem 3.1 and $A$ and $B$ have a unique common fixed point $x^{*}=0$.

Example 4.2. Let $X=[0,1]$ endowed with the $b$-metric:

$$
d: X \times X \rightarrow[0,+\infty), d(x, y)=|x-y|^{2}
$$

with constant $s=2$. Define mappings $A, B: X \times X$ by 
$A x=\frac{x}{16} \quad$ and $\quad B x=\frac{x}{8} . \quad$ Consider the mapping $\psi:\left(\mathbb{R}^{+}\right)^{9} \rightarrow \mathbb{R}^{+}$by

$$
\phi\left(x_{1}, x_{2}, x_{3}, x_{4}, x_{5}, x_{6}, x_{7}, x_{8} . x_{9}\right)=\frac{1}{9}\left(\sum_{i=1}^{9} \frac{x_{i}}{1+x_{i}}\right) .
$$

It is easy to verify that $(X, d)$ is a complete $b$-metric space and $A$ is continuous with respect to $d$. By calculus, we obtain that

$$
\begin{aligned}
& s^{6} d^{2}(A x, B y)=64\left(\left|\frac{x}{16}-\frac{y}{8}\right|^{2}\right)^{2} \\
& \leq 64\left(\frac{x^{2}}{256}+\frac{y^{2}}{64}\right)^{2} \\
& \leq \frac{7 x^{4}}{1536}+\frac{7 y^{4}}{256} \\
& \leq \frac{1}{9}\left(\frac{d^{2}(A x, x)}{1+d^{2}(A x, x)}+\frac{d^{2}(B y, y)}{1+d^{2}(B y, y)}\right) \\
& \leq \psi\left(\begin{array}{l}
d(A x, x) d(B y, y), d(A x, x) d(x, y), \\
d(B y, y) d(x, y), d(A x, B y) d(x, y), \\
d(A x, B y) d(A x, x), \\
d(A x, B y) d(B y, y), \\
d^{2}(A x, x), d^{2}(B y, y), d^{2}(x, y)
\end{array}\right) .
\end{aligned}
$$

That is, the contraction condition (11) holds. Theorem 3.3 ensures that $A$ and $B$ have a unique common fixed point $x^{*}=0$.

\section{Data Availability}

No data were used to support this study.

\section{Conflicts of Interest}

The authors declare that they have no conflicts of interest regarding the pub-lication of this paper.

\section{Authors Contributions}

All authors contributed equally and significantly in writing this article. All authors read and approved the final manuscript.

\section{Acknowledgments}

The second author would like to acknowledge the grant: Science and Research Project Foundation of Liaoning Province Education Department LQN201902 and the Research Foundation for the Doctoral Program of Shenyang Normal University BS201703, for financial support.

\section{References}

[1] S. Banach, Surles operations dans ensembles abstraits et leur application aux equations integrales, Fundamenta Mathematicae 3(1922), no.3, 51-57.

[2] J. Caristi, Fixed point theorems for mapping satisfying inwardness conditions, Trans. Am. Math. Soc. 215(1976), 241-251.

[3] T. L. Hicks, Fixed point theorems for quasi-metric spaces, Math. Jpn. 33(1998), no. 2, 231-236.

[4] I. Altun, G. Durmaz, Some fixed point results in cone metric spaces, Rend. Circ. Mal. Palermo 58(2009), 319-325.

[5] B. Choudhury, N. Metiya, Coincidence point and fixed point theorems in odered cone metric spaces, J. Adv. Math. Stud. 5(2012), no. 2, 20-31.

[6] I. Altun, H. Simsek, Some fixed point theorems on dualistic partial metric spaces, J.Adv. Math. Stud. 1(2008), 1-8.

[7] H. Aydi, Fixed point results for weakly contractive mappings in ordered partial metric spaces, J. Adv. Math. Stud. 4(2011), 1-12.

[8] A. Khan, M. Abbas, T. Nazi, C. Lonescu, Fixed points of multivalued contractive mappings in partial metric spaces, Abstr. Appl. Anal. 2014(2014), Article ID 230708.

[9] W. Shatanawi, A. Pitea, Fixed and coupled fixed point theorems for omega-distance for nonlinear contraction, Fixed Point Theory Appl. 2013(2013), Article ID 275.

[10] S. Czerwik, Contraction mappings in b-metric spaces, Acta. Math. Inform. Univ. Ostrav. 1(1993), 5-11.

[11] M. Boriceanu, Strict fixed point theorems for multivalued operators in b-metric spaces, Int. J. Mod. Math. 4(2009), 285-301.

[12] M. Boriceanu, Fixed point theory for multivalued generalized contraction on a set with two b-metrics, Stud. Univ. Babes-Bolyai Math. LIV, 2009.

[13] H. Aydi, M. Bota, S.Moradi, A common fixed points for weak b-contractions on b-metric spaces, Fixed Point Theory 13(2012), 337-346.

[14] V. Berinde, Generalized contractions in quasimetric spaces, Seminar on Fixed Point Theory Preprint 3(1993), 3-9.

[15] M. Pacurar, A fixed point result for qcontractions and fixed points on b-metric spaces without the boundness assumption, Fasc. Math. 43(2010), 127-136.

[16] W. Shatanawi, A. Pitea, R. Lazovic, Contraction conditions using comparision fuctions on $b$-metric spaces, Fixed Point Theory Appl. 2014(2014), Article ID 135.

[17] N. Hussain, M. Shah, KKM mappings in cone b-metric spaces, Comput. Math Appl. 61(2011), 1677-1684.

[18] S. Singh, B. Prasad, Some coincidence theorems and stability of iterative proceders, Comput. Math. Appl. 55(2008), 2512-2520.

[19] J. Matkkowski, Fixed point theorems for mappings with a contractive iterate at a point, Proc. Amer. Math. Soc. 62(1977), 344-348.

(C) The Author(s) 2019. This article is an open access article distributed under the terms and conditions of the Creative Commons Attribution (CC BY) license (http://creativecommons.org/licenses/by/4.0/). 